\title{
Neutron Diffraction Studies of a Fully Asymmetric Diffraction Geometry of a Bent Perfect Crystal with the Output Beam Compression
}

\author{
P. MikUla ${ }^{a, *}$, M. VRÁNA ${ }^{a}, J_{\text {S. ŠAROUN }}^{a}$, V. EM ${ }^{b}$ AND J. ČAPEK ${ }^{c}$ \\ ${ }^{a}$ Nuclear Physics Institute ASCR, v.v.i., 25068 Řež, Czech Republic \\ ${ }^{b}$ National Research Centre "Kurchatov Institute", Moscow, 123182, Russia \\ ${ }^{c}$ Faculty of Mathematics and Physics, Charles University, 12116 Prague, Czech Republic
}

\begin{abstract}
In this paper studies of neutron diffraction properties of the double crystal $(+n,-m)$ setting of a bent perfect crystal $\mathrm{Si}(311)$ in the fully asymmetric diffraction geometry with the output beam compression and the bent perfect crystal $\mathrm{Si}(220)$ in a symmetric diffraction geometry, are presented. The properties of the $(+n,-m)$ setting were studied for different curvatures of the individual crystal slabs. It has been found that after a beam condensation this fully asymmetric diffraction geometry can provide a monochromatic beam of a small width and of a practical use.
\end{abstract}

DOI: $10.12693 /$ APhysPolA.130.1114

PACS/topics: 03.75.Be, 25.40.Dn,

\section{Introduction}

An interest in fully asymmetric diffraction (FAD) geometry started in the past when elastically bent perfect crystals (BPC) have been found as a good alternative to conventional mosaic crystal monochromators or analyzers and several papers have been published on this topic [1-10]. In practice, the FAD geometry can be effectively studied in the case of BPC Si slabs, because the attenuation of thermal neutrons in $\mathrm{Si}$ is very low. First investigations of possible beneficial effects of the FAD crystal geometry were carried out within its employment in the nondispersive $(1,-1)$ double-crystal setting. In the first paper [1], calculations of the peak and integrated reflectivities as well as the effective mosaicity of the BPC elements including FAD geometry were introduced. In this paper also a highly efficient double-crystal monochromator based on two BPC Si slabs of the same cut, both in the FAD geometry and in the nondispersive setting $(1,-1)$, was proposed. The related results of the experimental test of such a double BPC monochromator were then presented in the next paper [2]. After that also one BPC slab of Si in the FAD geometry with the wide incident beam (several centimetres) and condensed output monochromatized beam was succesfully tested as a single monochromator for thermal neutrons [3-5].

In all mentioned cases, the resolution in $\Delta \theta$ or $\Delta \lambda / \lambda$ was comparable to that corresponding to the conventional mosaic monochromators/analyzers. After that, bent perfect crystals in the FAD geometry were tested as thermal neutron analyzers and succesfully used in nondispersive double crystal settings for high resolution small

\footnotetext{
* corresponding author; e-mail: mikula@ujf.cas.cz
}

angle neutron scattering (SANS) diffractometers [6-8]. A unique proposal of a possible use of FAD BPC Si slab as a high resolution TOF analyzer of thermal neutrons was also succesfully tested $[9,10]$. Finally, it should be mentioned that in the paper [11] the optics of double bent crystal rocking curves is theoretically described for symmetric and asymmetric diffraction geometries of the individual crystal slabs in a general mutual setting. Contrary to our earlier investigation of the properties of the BPC Si crystal in the FAD geometry with the output beam expansion [12], in the present paper we deal with the studies of the FAD geometry with the output beam compression. The FAD crystal is employed as analyzer in the $(+n,-m)$ double-crystal setting.

\section{Reflecting properties of the bent perfect crystal}

Bent perfect crystals (BPC) employed in a symmetric or an asymmetric diffraction geometry are well known for their focusing properties in real as well as momentum space with respect to a sample. However, in the limiting case of the FAD geometry as studied in the present case (see Fig. 1), one can hardly expect typical focusing properties. Generally, the BPC elements are attractive for an employment from the following reasons: predictability and reproducibility of the effective mosaicity and of a

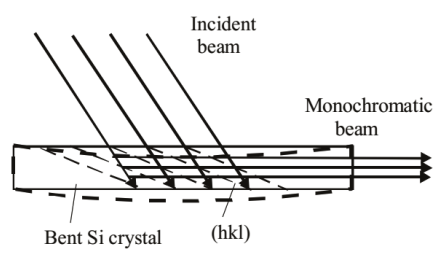

Fig. 1. Schematic sketch showing the FAD geometry of the Si crystal slab as used in the experiment. 


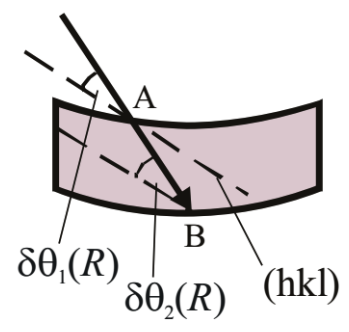

Fig. 2. Detail of the incident beam passing through the BPC slab.

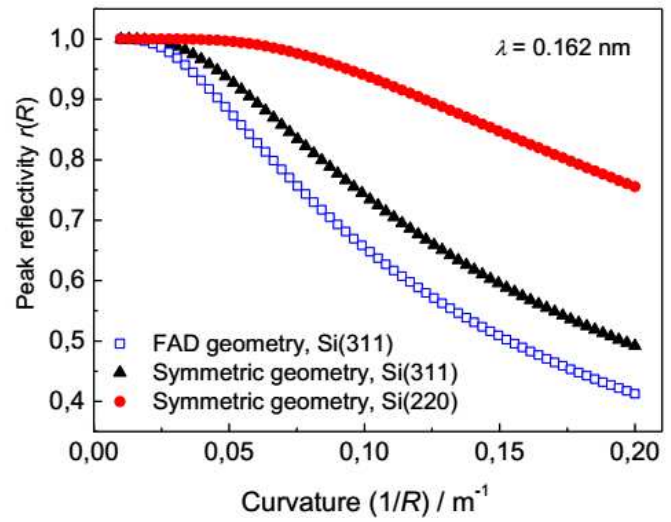

Fig. 3. Peak reflectivity vs. crystal curvature as calculated according to formulae (1) and (3) for symmetric $\mathrm{Si}(220)$, FAD (311) diffraction geometry and symmetric $\mathrm{Si}(311)$ for the sake of comparison.

rather high peak reflectivity uniformed over large areas of the crystal as well as of the integrated reflectivity. In the transmission geometry one can also benefit from natural wavelength focusing. The general formula for the peak reflectivity (reflection probability of a neutron fulfilling the Bragg condition) of neutron passing through a homogeneously deformed perfect crystal has been derived in a form as [13]:

$$
r(R)=\left\{1-\exp \left(-Q_{h k l}(\partial \Delta \theta / \partial s)^{-1}\right)\right\},
$$

where $(\partial \Delta \theta / \partial s)$ is the rate of change of the Bragg angle $\theta$ on the flight path $\Delta s$ in the crystal in the incident beam direction and $Q_{h k l}=\left(F_{h k l}\right)^{2} \lambda^{3} /\left(\Omega^{2} \sin 2 \theta\right)$ is the kinematical reflectivity of the crystal unit volume, where $F_{h k l}, \lambda$ and $\Omega$ are the structure factor, neutron wavelength and the unit-cell volume, respectively [14]. For cylindrically curved monochromator, the factor $\partial \Delta \theta / \partial s$ has been derived in general form [1]:

$$
\begin{aligned}
& \partial \Delta \theta / \partial s=(1 / R)(\cos \psi / \cos \theta) \\
& \quad \times[1-(1+\sigma) \sin (\theta+\psi) \sin (\theta-\psi)],
\end{aligned}
$$

where $\sigma$ is the Poisson constant and $\psi$ is the angle of asymmery. For FAD geometry $\psi=\theta_{0}$. Then the effective mosaicity $\delta \theta=\delta \theta_{1}-\delta \theta_{2}$ (see Fig. 2) and the integrated reflectivity $\rho^{\theta}(R)$ can be defined as $\delta \theta(R)=$ $\Delta s(\partial \Delta \theta / \partial s)$ and $\rho^{\theta}(R)=\delta \theta(R) r(R) A(\mu)$, respectively $(\Delta s=D / \sin (\theta+\psi)$, where $D$ is the thickness of the crystal slab and $A(\mu)$ is the attenuation factor). Then, for
FAD geometry we arrive at simple expressions for the effective mosaicity $\delta \theta(R)$ and the peak reflectivity $r(R)$ as $\delta \theta(R)=1 / R$ and $r(R)=1-\exp \left(-Q_{h k l} R\right)$, respectively. Figure 3 shows the dependence of the peak reflectivity $r(R)$ on the crystal curvature $(1 / R)$. It can be seen from Fig. 3 that the peak reflectivity corresponding to FAD geometry is slightly smaller than the one corresponding to the symmetric diffraction geometry. As to the attenuation factor $A(\mu)$, then in our case of low absorbing Si crystal, we can simply use the approximation expression $A(\mu)=\exp (-\mu L / 2)$, where $L$ is the length of the crystal slab.

\section{Experimental results}

The experimental studies were carried out on the dedicated neutron optics diffractometer installed at the reactor LVR-15 in Rež and operating at the fixed neutron wavelength of $0.162 \mathrm{~nm}$. No-Soller collimators are used on the beam path from the reactor to the detector. The schematic sketch of the first experimental performance is shown in Fig. 4. It can be seen from Fig. 4 that

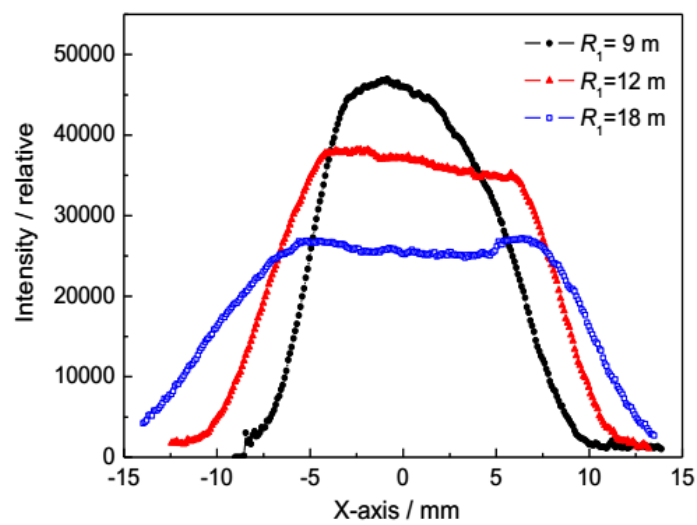

Fig. 4. Schematic sketch of the experimental arrangements as used for the studies of the FAD properties of the BPC Si (311) crystal in $(+n,-m)$ setting with respect to the $\mathrm{Si}(220)$ one. SC - scintillation camera was at $56 \mathrm{~cm}$ from the FAD crystal. Dimensions of the crystal slabs: $200 \times 40 \times 4 \mathrm{~mm}^{3}(l \times w \times t)$.

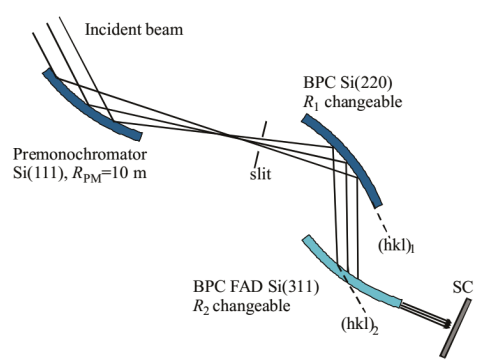

Fig. 5. Examples of the profiles of the beam coming from the bent $\mathrm{Si}(220)$ crystal for radii of curvature of 9 , 12 , and $18 \mathrm{~m}$. 


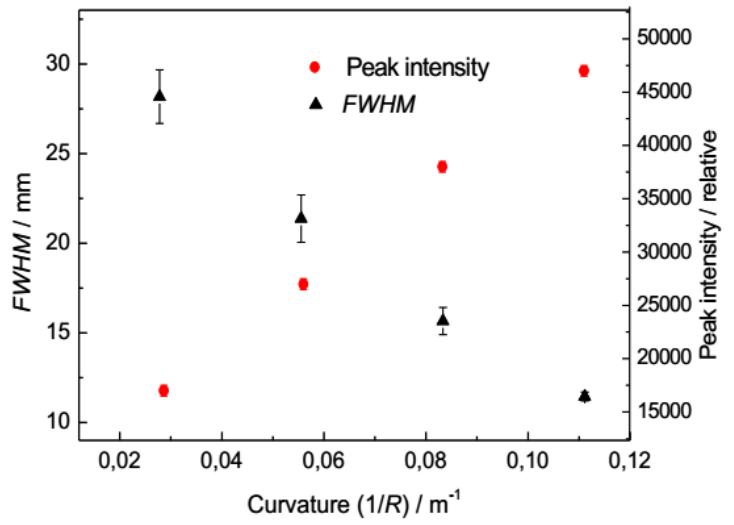

Fig. 6. FWHM of the incident beam coming from the BPC Si $(220)$ slab at the place just before the FAD BPC one.

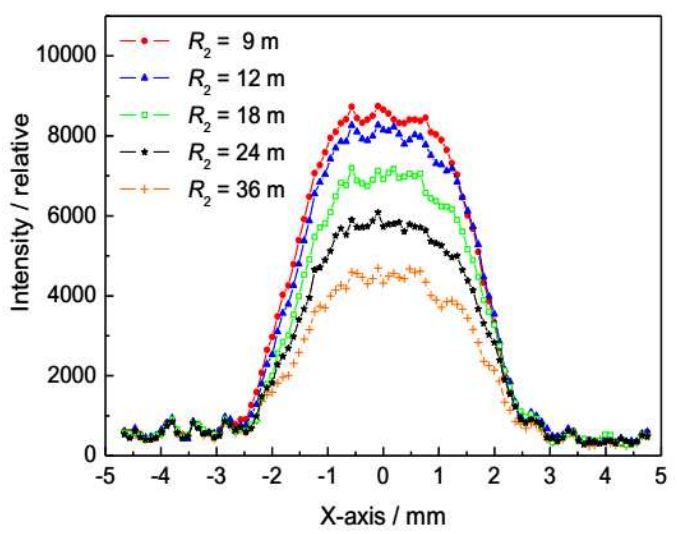

Fig. 7. Examples of diffraction profiles as registered by scintillation camera for $R_{1}=9 \mathrm{~m}$ and several values of $R_{2}$.

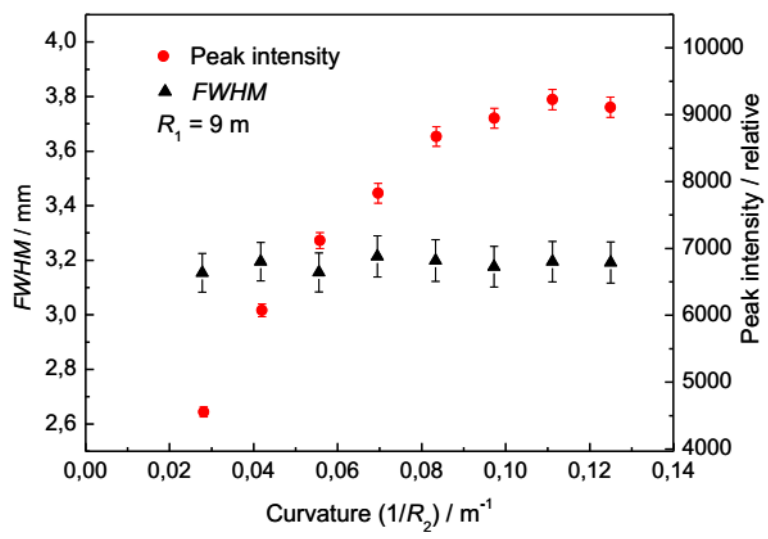

Fig. 8. Peak intensity and FWHM of the diffraction profile dependence on the FAD crystal curvature for a fixed curvature of $\operatorname{Si}(220)$ one of $0.11 \mathrm{~m}^{-1}$.

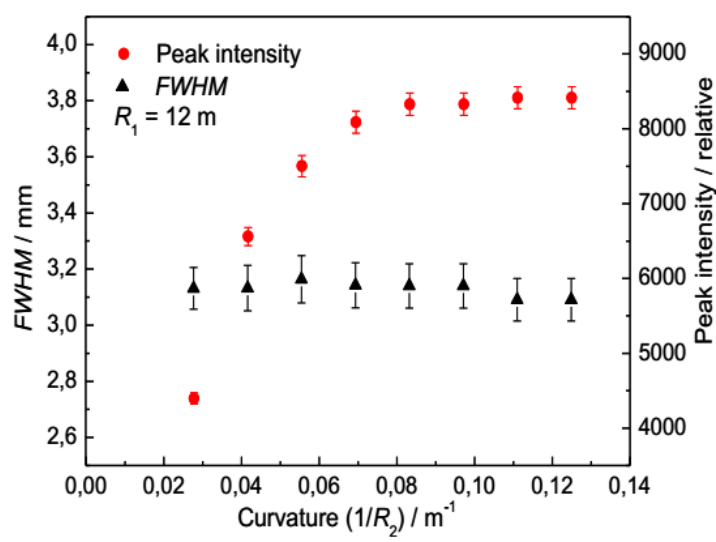

Fig. 9. As in Fig. 8, but for $\operatorname{Si}(220)$ curvature of $0.083 \mathrm{~m}^{-1}$.

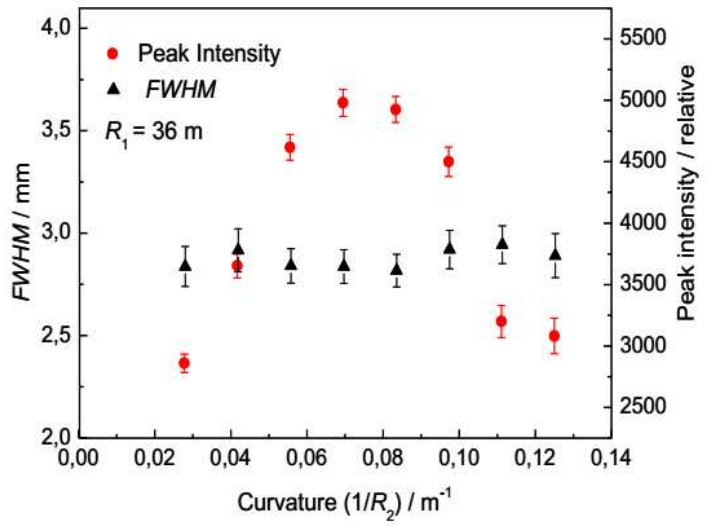

Fig. 10. As in Fig. 8, but for $\operatorname{Si}(220)$ curvature of $0.028 \mathrm{~m}^{-1}$.

the premonochromator with a small Bragg angle provides a monochromatic beam of a rather large divergency and $\Delta \lambda$ spread [14] which is then used for testing of the next dispersive double BPC setting. First, the profile of the beam incident on the FAD crystal was measured. The obtained results are shown in Figs. 5 and 6 . Then, as the curvature of both BPC slabs $\operatorname{Si}(220)$ and $\operatorname{Si}(311)$ were changeable, properties of the beam profile as registered by scintillation camera could be studied for different combination of the crystal curvatures. The obtained results are shown in Figs. from 7 to 10.

\section{Test of the focusing properties of the bent FAD crystal}

The test was carried out for some chosen curvatures of the $\operatorname{Si}(220)$ and FAD $\operatorname{Si}(311)$ crystal slabs (see Figs. 11 and 12). The inspection of Figs. 11 and 12 reveals that as has been expected, there is no special focusing on the beam diffracted by the FAD crystal which corresponds to the constant behaviour of FWHM in the dependence on the curvature $1 / R_{2}$. However, it should be pointed out that from the dependence of FWHM on the distance of the camera one can see that the beam diffracted by the 


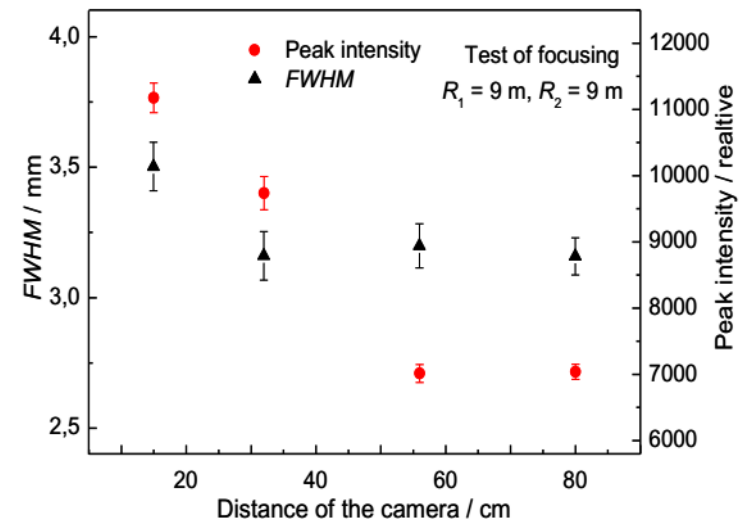

Fig. 11. Peak intensity and FWHM of diffraction profiles taken at different distances of the camera from the FAD crystal for $R_{1}=9 \mathrm{~m}$ and $R_{2}=9 \mathrm{~m}$.

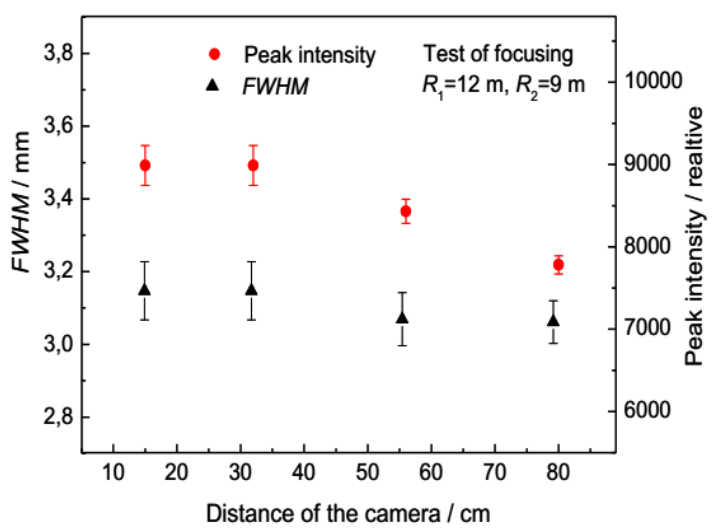

Fig. 12. As in Fig. 11, but for distances $R_{1}=12 \mathrm{~m}$ and $R_{2}=9 \mathrm{~m}$.

FAD crystal is compressed to the width slightly larger than $3 \mathrm{~mm}$ and also is rather perfectly collimated keeping the FWHM constant within the experimental errors at a large distance. This latter effect results from the fact that the bent FAD $\mathrm{Si}(311)$ crystal is in the dispersive setting with respect to the bent $\operatorname{Si}(220)$ one.

\section{Summary}

Some diffraction properties of the FAD geometry of the $\mathrm{BPC} \operatorname{Si}(311)$ slab in the $(+n,-m)$ double-crystal setting with respect to the $\mathrm{BPC} \operatorname{Si}(220)$ one were tested. Monochromatic beam provided by such a double-crystal setting is highly collimated and has a narrow width which is practically determined by the thickness of the FAD crystal. Due to the mutual dispersive setting of the BPC slabs, the double diffracted beam has also a narrow $\Delta \lambda$ spread of the order $10^{-3}$ or less. The peak reflectivities of the FAD $\operatorname{Si}(311)$ crystal and $\operatorname{Si}(220)$ crystal are for the used curvatures larger than 0.6 and 0.9 , respectively, the integrated reflectivity of the double-crystal setting corresponds to the overlapping of the individual phase space elements of the BPC Si(220) and FAD BPC Si(311) crystals. The presented diffraction performance can be practically exploited for high resolution powder diffraction, namely at high-flux neutron sources.

\section{Acknowledgments}

The related measurements were carried out at the CANAM infrastructure of the NPI ASCR Rez. The Bragg diffraction optics investigations are in the Czech Republic supported by GACR project no. 14$36566 \mathrm{G}$.

\section{References}

[1] P. Mikula, J. Kulda, M. Vrána, B. Chalupa, J. Appl. Crystallogr. 17, 189 (1984).

[2] P. Mikula, B. Chalupa, J. Kulda, M. Vrána, L. Sedláková, R. Michalec, J. Appl. Crystallogr. 18, 135 (1985).

[3] P. Mikula, J. Kulda, L. Horalík, B. Chalupa, P. Lukáš, J. Appl. Crystallogr. 91, 324 (1986).

[4] P. Mikula, P. Lukáš, R. Michalec, J. Appl. Crystallogr. 20, 428 (1987)

[5] P. Mikula, E. Krüger, R. Scherm, V. Wagner, J. Appl. Crystallogr. 23, 105 (1990).

[6] P. Mikula, P. Lukáš, F. Eichhorn, J. Appl. Crystallogr. 21, 33 (1988).

[7] J. Šaroun, P. Lukáš, P. Mikula, P. Strunz, J. Phys. Coll. C7 3, 439 (1993).

[8] P. Lukáš, P. Mikula, J. Šaroun, P. Strunz, Nucl. Instrum. Methods Phys. Res. A 338, 111 (1994).

[9] P. Mikula, M. Vrána, M. Furusaka, Y. Yasushige, in: Proc. Int. Conf. ICANS XVI, Eds. G. Mank, H. Conrad, Düsseldorf-Neuss (Germany) 2003, p. 401.

[10] P. Mikula, M. Vrána, M. Furusaka, V. Wagner, Y.N. Choi, M.K. Moon, V.T. Em, C.H. Lee, Nucl. Instrum. Methods Phys. Res. A 529, 138 (2004).

[11] M. Popovici, A.D. Stoica, B. Chalupa, P. Mikula, J. Appl. Crystallogr. 21, 258 (1988).

[12] P. Mikula, M. Vrána, B.S. Seong, W. Woo, V. Em, D. Korytár, J. Phys. Conf. Series 528, 012004 (2014).

[13] J. Kulda, Acta Crystallogr. A 40, 120 (1984); Acta Crystallogr. A 44, 283 (1988).

[14] G.E. Bacon, Neutron Diffraction, Clarendon Press, Oxford 1975, p. 67 and p. 95. 